\title{
Healthcare recommender system based on Smart-health Routes
}

\author{
${ }^{1}$ M. Sandeep Kumar , ${ }^{2}$ Prabhu. J \\ ${ }^{1}$ Research Associate, Department of School of Information Technology, \\ VIT University, Vellore-632014, TamilNadu, India. \\ 2 Associate Professor, Department of School of Information Technology, \\ VIT University, Vellore-632014, TamilNadu, India \\ sandeepkumarm322@gmail.com, j.prabhu@ vit.ac.in
}

\begin{abstract}
Recommender systems have proved more significant area of research and outstanding to the technology progress in mobile devices like smartphone and tables. Mobile technology is grown quickly due to social networking. The concept of a recommender system to provide item feedback to the user in which it makes a decision based on the rating of an item purchased by the previous user. This paper describes regarding Content -ware recommender system in offering a personalized recommendation for exercise routes to people based on their health condition in which real-time data depends on the city they lived. Finally, we discuss challenges and Opportunities play in context-aware recommendation system.
\end{abstract}

Keywords - Context-aware recommendation, Smart city, Routes. Healthcare.

\section{I.INTRODUCTION}

Currently most of the person facing issues in medical and healthcare. Many people suffer from chronic attack arthritis and back pain [25], due to improper exercise and diet [1].Moreover, additional things like air pollution affect illness, premature deaths annually around the world [2].Air pollution produces more harms to the health of children, elderly and people due to chronic diseases[3,25].Each Individual lifestyle can help to decrease the physical issues like cardiorespiratory, musculoskeletal and morbidity [4,25].Further, it has definite neurocognitive causes[5].For example, improvement of cardiorespiratory exercise related with cortical thickness in adult health and elder diagnosed with dementia[6].Hence maturity of security required to follow a healthy lifestyle could be a more challenging task for public health care system. Healthcare domain has overcome this issues has attempted to combine with new innovative technologies that encourage a healthy lifestyle. While the distribution of those service show is starting stage of investment, in long-term and follow to decreased healthcare system based on cost, of improvement made by people in the lifestyle.

Most healthcare model is frequently shifting to patientcentric approaches. In this method, patients not only have passive elements but it also has individual contributors of their own in associates with health [25]. Some more broad perspective in sharing the known information to other that leads to recommender systems [7].It involved with combined transparently of our daily lifestyle. This system put the effect in collaboration between users in support to produce better decisions. In advance, the introduction of augments mobile health in the context of smart health [8, 25].It provides suggesting routes for doing exercise without any issues in real time by utilizing context-aware recommender system and mobile applications [25].

\section{II.RELATED WORK}

Bernd blobel et al. [9] describes context-aware recommender system for improving daily health activity. The system will follow user's information, interest, time, location and regular exercise. The method illustrates a performance context aware-recommender system and context model of health improvement presented in a recommender system. Ekiyot Kaur et al. [10] demonstrate a user context model based on usability attributes for implementing the mobile application in healthcare domain. We need to pick specific characteristics in combined with a mobile app to offer usercentered and context ware procedure. This system evaluated based on the user doing peritoneal dialysis (PD) in mobile; it has tested with real users.

Andre c.M .costa et al. [11] represent action between recommender system and context-aware computing in implement [27] with COReS (Context-aware, an ontologybased recommender system for service recommendation).It can infraware context-aware environment in producing services to a system like personalized and proactive.Agusti Solanas et al. [8] demonstrate a new concept of smart health; it can use in content-aware recommendation system on mobile health among smart cities. This system offers an overall representation of intelligent health, challenges, and opportunities for further research in recommendation system. Sergio Ilarri et al. [12] describe various types on the sensor used to find accuracy in the recommendation in different field like healthcare, e-learning, mobile computing and also discuss open issues in context-aware recommendation system while using sensors.

Mdlleas pramanik et al. [13] demonstrates big data enabled smart healthcare system framework (BSHSF) [26], it provides 
intellectual formation of both intra and inter Organisation of the business model in healthcare domain. We also illustrate various challenges and opportunities presented in BSHSF to healthcare domain. BSHSF applied based on five recommendations for performing adequately in the healthcare sector.Hanna Schafer et al. [14] discuss overview about process towards recommendation system in identifying, personalized and complex medical intervention, health measures. We also find some key challenges of decision support system that required in high risk for health care.

\section{A) TOWARD SOCIAL AWARE COMMODITY}

Most of the health-related mobile application based on sports and health monitoring. Currently, the survey shows that fast development in healthcare application and ambient assisted living solutions [15].This type of growth in demand and system leads to the notion of context awareness to aware social process [16].Moreover, extensive data's has collected from a heterogeneous system that leads to new collective intelligence. Hence users turn as part of large urban superorganisms [17].Finally, it moves towards the next generation, trends and solution applied in context-aware technology. For illustrating those, we used sensing infrastructure for smart cities and collective action for crowdsourcing of information [25].

\section{B)RECOMMENDER SYSTEM AND COLLABORATIVE FILTERING}

Most of the recommender system involves in the domain of knowledge discovery databases, in which organization utilizes to discover a pattern in the last gathering of data. Moreover, in a typical recommender system, people recommend input, then system aggregate to direct the correct recipients [18, 25].More specifically; collaborative filtering produces a recommendation on a set of an item like books, music, films, health, and routes, based on user choice [25]. The set of users will select elements based on rating preferred by the previous user $[19,21]$ Collaborative filter trusts only large database for user evaluations. The primary focus $\mathrm{CF}$ recommendations on assumption of similarity user interest on same items.

CF methods are categories, allow to the data they utilize

Memory-based methods: This method uses the data matrix of all entries, rating, and relationships.

Model-based methods: It calculates the statistical models and functions based on data matrix.

Hybrid methods: This method integrates with previous two methods with content-based information to improve recommendations. The proposed frameworks focus on memory-based methods that appliedto neighborhood search. It aims to find a group of similarity, preference, prediction by performing various techniques in recommendation system.

\section{III.PROPOSED WORK}

\section{HEALTH ROUTES TO USERS}

Most of the people take part of individual lifestyle due to medical recommendations based on the occurrence of health issues. Hence, this activity usually towards more emotions and few facts. Mostly this approach applies to the people for selecting activities like sports, exercise but they do not have proper guidance to follow and perform it. For example, jogging may be more beneficial for a person to determine the place to play it. Most of the people used to prefer it based on distance and weather. Some avoided factors while jogging like air pollution, crown density; it may lead to chronic disease for people.

For solving this type of issues, our approach provides realtime constraints and information from various sources namely real-time data from smart city infrastructure [25], usage of routes for people based on health condition. The system has some features like i) It used to prefer routes based on best-fit of user's requirement and preferences. ii) Users can apply with smartphones. iii) The System will offer both dynamic and collaborative in following the real-time modification. It consequence depends on variable monitoring by city sensor iv) Users perform in sensor that provides information and experience to the systems. The user may inform the policy regarding the unexpected condition that may affect other users.vi) the user can show new routes, to enrich the system.

Context-aware recommender system software performs based on servers and preference of routes. The recommendation system involved main procedure of five-step; each corresponds to the numbers in fig 1 .

i) The user provides bidirectional interaction with contextaware recommendation system to ask regarding recommending routes offered in the system. User duty to send information about his/her location.

ii) Smart city's communication infrastructure acquired by real-time environment data is namely air quality, ultraviolet radiation, wind speed, temperature, and precipitation from sensors and transfer the data to contextaware recommendation system. This system estimates the information from the nearest sensor from individual routes in data storage known as matrices. The system also allocated based on three status to routes

Danger- The path mayoffer harmful to the user's health. It will not be suitable for individual users.

Caution- Potential risk exists, for example, some user follows more environmental factors.

Idle- No proof of risk exists.

Context-aware recommendation system analyzes crowdsourced information when performing route's status.

iii) The system will check user's preference and employ a collaborative filter to the entire database of routes for decreasing the top $\mathrm{N}$ preference. iv) The system utilizes 
healthcare data about user and information obtained from smart city to avoid danger routes, before cause harm to users. It automatically allocates "Caution "signal to routes with much indentation about users. After employ $\mathrm{CF}$, if no route satisfies this case, system get back to the third step in performing a new set of recommendation. In the end, the system transfers the user a list of the $\mathrm{N}$ routes.Fig2 shows a description of Context-aware recommendation system decision flow or workflow. They have set of procedure to run until system prefers best fit to the user based on user's health condition and environment constraints.

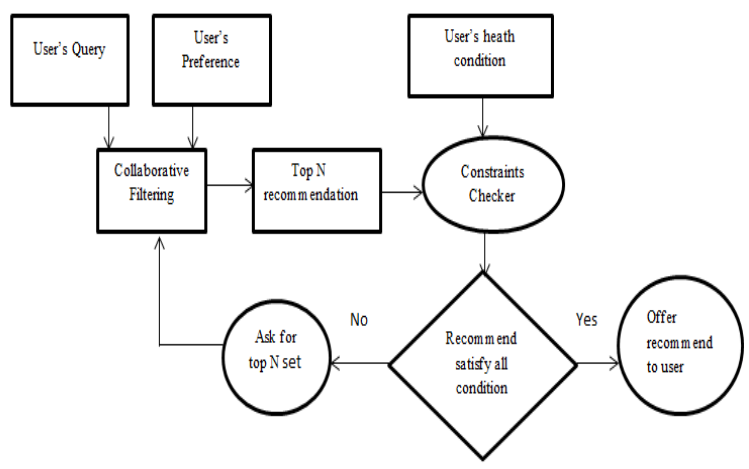

Fig 1: System architecture of content-aware recommendation system

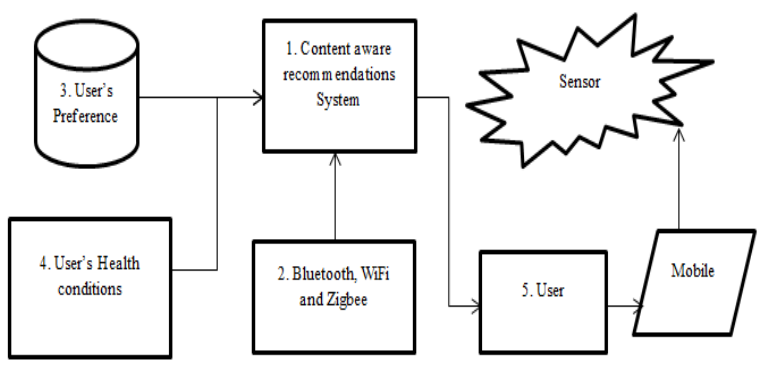

Fig2: Shows description of Context-aware recommendation system decision flow.

\section{SMART ROUTES}

Users can communicate with context-aware recommendation system by smart route mobile app that available in google store. This app used to view real weather data and air quality estimation form Catalan air -quality monitoring system.

i) Users can log into this app from home screen; we can view the closest route, specific route, top route. We need to create a route by checkpoints while walking.

ii) The app menu shows the users check and maintain their records from various route sessions and update users' health condition.

iii) The user is required to review whether and air quality formation in routes.

iv)The user needed to provide feedback regarding app(Like suggest in changing color and offer quality information.

v)Once the user selects a route, that application will display the starting point and destination point.

vi)Distance, duration, speed, alert and change in route. vii) The user can send an alert message regarding issues in route.
Finally, the app shows an overall statistical collection during the process of sessions that utilized by users.

\begin{tabular}{|l|l|l|}
\hline Dataset & Users & Routes \\
\hline Tarragona & 2000 & 11 \\
\hline Barcelona & 10000 & 28 \\
\hline
\end{tabular}

Table 1: Description of dataset in term number of users and route

\section{RESULT AND DISCUSSION}

To demonstrate this approach can perform two datasets from different cities like Tarragona and Barcelona datasets. The Tarragona dataset consists of 22 routes and, 2000 user and Barcelona dataset comprised of 28 routes and 10000 users in table 1. We construct the simulate users based on age and medical statistics form world health Organisation and world heart federation. User profiled based on health issues, and content aware recommendation rating routes depend on user's health report and features of routes. Further, we show technical documents: Implementation and validation of smart health applications [20].

By using those two datasets, we estimate our approach based on accuracy and robustness with sparse data. i)we perform for 10 to $50 \%$ of rating that stored in the datasets. ii) A predicate that evaluation by $\mathrm{K}$-nearest neighbor approach. By employing this approach with $\mathrm{k}=1$. while, we could have utilized large values of $\mathrm{k}$ using $\mathrm{k}=1$ in decreasing the performance cost in practical. We also examine neighbors by using the Euclidean distance between used and control of same routes. Finally, estimating the MAE (mean absolute error) among original values and the value that procedure estimate.

From Tarragona datasets, we found that MAE of 12.20 with $10 \%$ of the undefined data and only 13.89 MAE with $50 \%$ of data. We know what percentage of unknown data is more, so it has an error, but still, it remains as low levels in satisfying the user preference. In Barcelona datasets, MAE growing slow and value at a lower level. Hence it requires more referrals for more chances of identifying the similar users. Finally, we perform with the trial with 20 real users in Tarragona using the smart route to repository user preference and offer a recommendation. Being of small sample size, we find more difficult in identifying similarity users .when improving the amount of unknown data. A further suggestion showed accurate and more MAE below 17, $81 \%$. By comparing that two datasets, found that simulated data's are similar to those with real data.

\section{CHALLENGES AND OPPORTUNITIES}

\section{i) Challenges}

The concept of smart health performs independently in healthcare domain. The Certain parameter requires following by user in smart health namely technological, financial, logistic and psychological needs [8, 22]. Here some of the 
leading challenges that are examined and shown in smart health to overcome those things.

\section{A) Multidisciplinary research interaction}

Most of researcher and practitioners figure out in the same Organisation; it is hard to share knowledge among them that leads to interdisciplinary solutions [8]. The conceptuality of shealth based on communication and collaboration between many actors like government, researchers, physicians and practitioners to adopt ordinary ground from starting the level, hence to neglect unnecessary design and over-spending.[8,21] B) Sensor integration

One significant challenge is facing during implementation process in ambient intelligence. Ambient intelligence helps the sufficient account for co-existence in theheterogeneous system [8,21].It's performance based on topological and morphological dependence from the location at which it operates[21].Especially this argument will be right in case of dense urban situations, in which radio-electric affect provided by multipath propagation[8]. In some scenario, body area network [23] has a component of complexity that adds due to the impact of the human body presents in the wireless channel. Further privacy issues in urban sensing ecosystem need to address partially $[8,24]$.

\section{C) Big data management and cloud}

In general, data classified into 3v's namely volume, velocity, and variety. In a smart city, those $3 \mathrm{v}$ 's plays a crucial role in the context of smart health. Variety- data collected by from sensors namely temperature, pollution, and allergens[8]. Velocity-data needs to collect and examine in real-time offered by users. Volume-Large amount of data gathered from sensors has measured in every second. While managing large data's from real time, we are facing specific issues in storage and bandwidth. A particular way to approach this issue by including the cloud paradigm. Moreover, it will not be a direct method to implement this solution. Due to scalability, we have many implications for privacy, security, multi-tenancy, assess control, and so forth [8].Some more challenges in mining and analysis the large data with artificial intelligence and statistics communities [8].

\section{D) Usability and human interaction}

How the user will communicate with the city is still an open problem that needs to be resolved. Design improved wearable, unobtrusive sensor, compactness, weight, autonomy, reliability is some problems need to be noted. To address those problems, we need succeed in smart cities and health.

E)Others- Further Postolache et al.[22] finds some more challenges in following pervasive healthcare such as financial constraints, the complexity of technical challenges, Organisation issue, a collaboration between entire stakeholders and describing the proper quality audit stage and culture process.

\section{ii) Opportunities}

Some open opportunities while developing new health-related application and services.[20,21]

A) Data collection, presentation, and analysis
The Real-time data are gathered from patient and integrated within public data. Routes, health records are combined with data to define sensors, cameras, weather report, and forecasts. $\mathrm{B})$ Engaging patient and families in management

In smart health, users play a significant role in empowering and efficient in supporting the active user to maintaining their health. The system can use for medical data and offer guidance for action; habit made every day inside the city. For example, smart health application may givespatients with storage issue with the best route by neglect areas consider as more atmospheric pollution.

\section{C)Improving policy decisions}

Smart health system may assist public health Organisation. In both policies and decisions have personalized to the city, districts population, health hazards, climate, environment, and infrastructure [8]. Most of the opportunities arise quickly due to mining data in associate with best public health decision making[8].

\section{D) Epidemic control}

Both smart health data and its methodology improve quickly due to its efficiency in detecting and access the epidemics. Location and actions can be used to identify essential new scenario during the epidemic, active finding areas for improving risk and optimal to maintaining a raging epidemic. Hence this methodology can employ to recognize and manage another unreasonable health risk like pollution radiation from industry.

\section{E) Cost saving}

The entire previous analysis, sector may have a substantial effect on health care in reducing cost. Cost reduction may associate with improving the system efficiency in offering services [8]. The Optimized disease management and prevention may lead to decrease the unwanted hospital view and sharp events from poorly maintained chronic patients. Moreover reduction time for action and efficient public health management may offer optimal result; meanwhile, it decreases cost worldwide [8].

\section{V.CONCLUSION}

Recommender system plays a crucial role in current medical research. Due to improvement in mobile technology and social network, it is effortless for the user to utilize recommender system efficiently. This paper describes regarding Content -ware recommender system in offering a personalized recommendation for exercise routes to people based on their health condition in which real-time data depends on the city they lived. Experiment analysis based on two datasets for estimating accuracy and robustness with sparse data. Most predominant used in medical research is $\mathrm{K}$ nearest neighbors approach.It examines nearest neighbors by utilizing Euclidean distance between user and control of same routes. The system estimate based on a mean absolute error(MAE) between the original value and the value that procedure estimates. Finally, discuss challenges and opportunities play in context-aware recommendation system 
We can perform with two types of users like i) one need to follow preference and other should avoid it. Long-term performance of data is from the user, smart city and health specialist to perform advantages of health activity in integrating with use of smart city information. We plan to offer personalized recommendations using fusing data with information linked from EHR (electronic health record).It required integrating medical research to define the baseline data needed from history to improve recommendations based on other activity than proposed work.

\section{VI.ACKNOWLEDGMENT}

A particular note of thanks to Vellore Institute of Technology (VIT) University for providing necessary infrastructure facilities to carry out the research work and my friends who have helped me to complete this study.

\section{REFERENCES}

[1] World Health Organization. (2013). Global action plan for the prevention and control of non-communicable diseases 2013-2020.

[2] World Health Organization. (2010). Preventing Disease through Healthy Environments-Exposure to Air Pollution: A Major Public Health Concern.

[3] Bogdanović, D., \& Lazarević, K. (2014). Early Warning System and Adaptation Advice to Reduce Human Health Consequences of Extreme Weather Conditions and Air Pollution. In Handbook of Research on Democratic Strategies and Citizen-Centered E-Government Services. IGI Global.

[4] Vos, T., Flaxman, A. D., Naghavi, M., Lozano, R., Michaud, C., Ezzati, M., \& Abraham, J. (2013). Years lived with disability (YLDs) for 1160 sequelae of 289 diseases and injuries 1990-2010: a systematic analysis of the Global Burden of Disease Study 2010. The Lancet, 380(9859), 2163-2196.

[5] Smith, J. C., Erickson, K. I., \& Rao, S. M. (2015). Introduction to the JINS Special Issue: Physical Activity and Brain Plasticity. Journal of the International Neuropsychological Society: JINS, 21(10), 743.

[6] Reiter, K., Nielson, K. A., Smith, T. J., Weiss, L. R., Alfini, A. J., \& Smith, J. C. (2015). Improved cardiorespiratory fitness is associated with an increased cortical thickness in mild cognitive impairment. Journal of the International Neuropsychological Society, 21(10), 757-767.

[7] Resnick, P., \& Varian, H. R. (1997). Recommender systems. Communications of the ACM, 40(3), 56-58.

[8] Solanas, A., Patsakis, C., Conti, M., Vlachos, I. S., Ramos, V., Falcone, F. \& Martinez-Balleste, A. (2014). Smart health: a context-aware health paradigm within smart cities. IEEE Communications Magazine, 52(8), 74-81.

[9] CERÓN-RIOS, G., LÓPEZ, D. M., \& Blobel, B. (2017, May). Architecture and User-Context Models of CoCare: A Context-Aware Mobile Recommender System for Health Promotion. In PHealth 2017: Proceedings of the 14th International Conference on Wearable Micro and Nano Technologies for Personalized Health 14-16 May 2017 Eindhoven, The Netherlands (Vol. 237, p. 140). IOS Press.

[10] Kaur, E., \& Haghighi, P. D. (2016, November). A Context-Aware Usability Model for Mobile Health Applications. In Proceedings of the 14th International Conference on Advances in Mobile Computing and Multi-Media (pp. 181-189). ACM.

[11] Costa, A., Guizzardi, R., Guizzardi, G., \& Pereira Filho, J. G. (2007). COReS: Context-aware, Ontology-based Recommender system for Service recommendation. In Proceedings of the 19th international conference on advanced information systems engineering (CAiSE'07) (pp. 11-15).

[12] Ilarri, S., Hermoso, R., Trillo-Lado, R., \& Rodríguez-Hernández, M. D. C. (2015). A review of the role of sensors in mobile context-aware recommendation systems. International Journal of Distributed Sensor Networks, 11(11), 489264.
[13] Pramanik, M. I., Lau, R. Y., Demirkan, H., \& Azad, M. A. K. (2017). Smart health: Big data enabled health paradigm within smart cities. Expert Systems with Applications, 87, 370-383.

[14] Schäfer, H., Hors-Fraile, S., Karumur, R. P., Calero Valdez, A., Said, A., Tarkanian, H. \& Trattner, C. (2017, July). Towards Health (Aware) Recommender Systems. In Proceedings of the 2017 International Conference on Digital Health (pp. 157-161). ACM.

[15] Rashidi, P., \& Mihailidis, A. (2013). A survey on ambient-assisted living tools for older adults. IEEE Journal of biomedical and health informatics, 17(3), 579-590.

[16] Lukowicz, P., Pentland, S., \& Ferscha, A. (2012). From context awareness to socially aware computing. IEEE pervasive computing, 11(1), 32-41.

[17] Zambonelli, F. (2012). Toward sociotechnical urban superorganisms. Computer, 45(8), 76-78.

[18] Resnick, P., \& Varian, H. R. (1997). Recommender systems. Communications of the ACM, 40(3), 56-58.

[19] Su, X., \& Khoshgoftaar, T. M. (2009). A survey of collaborative filtering techniques. Advances in artificial intelligence, 2009, 4.

[20] Casino, F., Patsakis, C., Martinez-Balleste, A., Borras, F., \& Batista, E. (2017). Technical Report: Implementation and Validation of a Smart Health Application. ArXiv preprint arXiv: 1706.04109.

[21] Casino, F., Patsakis, C., Batista, E., Borras, F., \& Martinez-Balleste, A. (2017). Healthy Routes in the Smart City: A Context-Aware Mobile Recommender. IEEE Software, (6), 42-47.

[22] Postolache, G., Girão, P. S., \& Postolache, O. (2013). Requirements and barriers to pervasive health adoption. In Pervasive and Mobile Sensing and Computing for Healthcare (pp. 315-359). Springer Berlin Heidelberg.

[23] Jovanov, E., \& Milenkovic, A. (2011). Body area networks for universal healthcare applications: opportunities and challenges. Journal of medical systems, 35(5), 1245-1254.

[24] De Cristofaro, E., \& Di Pietro, R. (2013). Adversaries and countermeasures in privacy-enhanced urban sensing systems. IEEE Systems Journal, 7(2), 311-322.

[25] https://www.computer.org/

[26] https://scholars.cityu.edu.hk/

[27] Otebolaku, A. M., \& Andrade, M. T. (2015). Context-aware media recommendations for smart devices. Journal of Ambient Intelligence and Humanized Computing, 6(1), 13-36. 